\title{
Le label : facteur d'internationalisation de la marque des coopératives marocaines
}

\author{
GHILANE Hind \\ ESLSCA Business School Rabat \\ MARIAM FILALI ALLACH \\ ELSCA Business School Paris - Campus Rabat
}

Résumé : «Les coopératives, des entreprises pour un monde meilleur », telle était la déclaration des Nations Unies dans leur vision de faire de l'année 2012 l'année internationale des coopératives (ONU, 2012). Cet intérêt vient répondre en faveur de la place occupée par cette structure dans le cadre du développement économique et social, et ce partout dans le monde. Au Maroc, le même intérêt est porté à ces types de structures. Face au contexte concurrentiel international, la coopérative doit développer un avantage concurrentiel inimitable: sa stratégie de marque. L'objet de cet article porte sur l'identification des facteurs qui ont un impact positif sur l'internationalisation de la marque coopérative marocaine. Une étude quantitative a été réalisée sur un échantillon de 200 personnes déterminées selon la méthode de convenance. L'échantillon est composé de citoyens français. La validation du modèle est faite par la méthode LISREL. Les principaux résultats indiquent que les variables qui ont le plus d'impact sur la réussite de la stratégie d'internationalisation de la marque de la coopérative marocaine sont la qualité et les labels.

Ainsi, les résultats de notre étude permettront aux managers de bien identifier les variables constitutives de leur stratégie de marque pour réussir son internationalisation.

Mots-clés : Stratégie de marque, Internationalisation, Coopératives.

Digital Object Identifier (DOI): https://doi.org/10.52502/ijesm.v1i2.226 


\section{Introduction}

La marque a connu une grande évolution au fil du temps, dans un premier temps, ses fonctions principales se limitaient à quelques fonctions qui tournent principalement autour de l'identification, de l'appropriation et de la différenciation. Aujourd'hui, on constate une nouvelle perception du rôle de la marque, fondée sur la garantie par la confiance, la fonction identitaire ou encore la fonction aspirationnelle.

Ce développement vient principalement satisfaire le marché de consommation, répondre à un consommateur de plus en plus exigeant et garantir une position sur un marché concurrentiel.

Les marques jouent un rôle important dans la détermination du positionnement. Ils sont décrits comme un avantage concurrentiel inimitable. Selon Porter (1985), l'avantage concurrentiel est ce qui différencie l'offre de base d'une entreprise de ses concurrents, et constitue donc son pouvoir différenciant. Un avantage concurrentiel doit être précieux pour être perçu par les consommateurs comme nettement supérieur, rare et difficile à imiter.

Plusieurs acteurs interagissent pour le développement de l'économie locale et internationale, entreprises, associations et coopératives. Notre recherche porte une attention particulière à la coopérative en tant que structure hybride qui combine deux composantes : l'entreprise et l'association. Nous prenons comme référence Prévost (2001), qui a eu le mérite de présenter la coopérative comme une structure hybride qui associe l'entreprise et l'association.

Certains auteurs ont soutenu que les coopératives ne sont pas séparées du phénomène de la mondialisation des marchés et de la concurrence Errasti, et al. (2003). En effet, la coopérative interagit dans le même environnement que l'entreprise et les autres structures, elle fait face aux mêmes contraintes de concurrence et d'ouverture des marchés. Dans cette perspective, la coopérative doit développer un avantage concurrentiel inimitable pour assurer sa présence tant sur le marché local que sur le marché international. Cet avantage concurrentiel se présente sous la forme de la marque.

Les chercheurs intéressés par les coopératives ont commencé à s'interroger sur l'efficacité des structures coopératives à générer des résultats durables, à s'orienter vers le marché et à créer et perpétuer des marques Beverland (2005), Cook et Iliopoulos (1999), Croompe et al. (1991). Cependant, les résultats ont révélé que pour de nombreuses coopératives, il y avait une reconnaissance tardive de la nécessité d'investir dans le marketing Beverland (2005), Edwards et Shultz (2005), mais l'intérêt est très présent. Selon Michel (2009), la marque représentée sous la fonction de garantie, identifiée comme fonction de confiance, est liée à la notion de label et de qualité.

\section{Le marketing mix de la coopérative}

Boisvert (1981), propose une définition précise du marketing coopératif, et le présente comme un équilibre qui doit être fait entre deux objectifs, l'un concernant la raison d'être de la coopérative et l'autre concernant la dimension économique.

Pour la coopérative en tant que système hybride, le programme de commercialisation doit respecter le principe de cohérence. Le produit doit répondre aux besoins des parties prenantes, à savoir les membres et les consommateurs.

La littérature présente la politique produit de la coopérative doit mettre en avant l'originalité, la typicité et l'authenticité de ses produits, tout en s'intéressant à l'aspect lié à l'innovation pour ancrer le produit dans l'authentique Fort (2006). Le consommateur a besoin de plus d'informations sur l'origine des produits, le processus de production et la traçabilité Rastoin (2004).

Quant au principe de la stratégie tarifaire, il doit être adapté au principe du commerce équitable Grandval, Soparnot (2005). La politique des prix devra répondre à des objectifs sociaux et économiques 
grâce à une rémunération correcte et satisfaisante des producteurs locaux pour améliorer les conditions de vie des producteurs Capron, Quairel (2002).

Pour les circuits de distribution, la coopérative a la possibilité de vendre dans ses propres locaux, ou de vendre sur des foires et marchés équitables, ou par un intermédiaire en évitant la sous-intermédiation en utilisant la livraison directe. Des détaillants via des représentations locales ou des grossistes, voire en recourant à des circuits spécialisés, à savoir des magasins spécialisés dans la vente de produits équitables Attouch (2014).

Selon Gouin et Perraud (2008), la communication dans une coopérative spécialisée dans la production de produits locaux doit se concentrer sur l'identité (origine, qualité) en s'appuyant sur les éléments suivants : authenticité, éthique, société ; origine : coopérative ; artisanat ; positionnement des produits : étiquette ; produits locaux ; qualités naturelles ou nutritionnelles.

Pour l'image de marque, les consommateurs, les changements de modes de vie, les problèmes de santé, les images positives du pays d'origine et les préoccupations environnementales ont permis à chaque responsable de marque de créer une identité de marque pour la coopérative autour de ces attributs Beverland (2007).

Tableau 1 : Principales variables du mix marketing des coopératifs

\begin{tabular}{|l|l|l|}
\hline Dimension & Variable & Auteur \\
\hline Produit & $\begin{array}{l}\text { Origine des produits, processus de fabrication } \\
\text { et traçabilité. }\end{array}$ & Rastoin (2004) \\
\hline Prix & $\begin{array}{l}\text { Atteindre les objectifs sociaux et } \\
\text { économiques. }\end{array}$ & Capron, Quairel (2002) \\
\hline Communication & $\begin{array}{l}\text { Authenticité, éthique, société ; origine : } \\
\text { coopérative ; artisanat ; positionnement des } \\
\text { produits : étiquette ; produits locaux ; qualités } \\
\text { naturelles ou nutritionnelles. }\end{array}$ & Perraud (2008) \\
\hline Distribution & $\begin{array}{l}\text { Vendre dans ses propres locaux, ou vendre } \\
\text { dans les foires et marchés équitables, ou par } \\
\text { un intermédiaire. }\end{array}$ & Attouch (2014) \\
\hline Marque & $\begin{array}{l}\text { Changements dans les modes de vie, } \\
\text { problèmes de santé, images positives du pays } \\
\text { d'origine préoccupations } \\
\text { environnementales. }\end{array}$ & Beverland (2007) \\
\hline
\end{tabular}

Source : Réalisé par l'auteur

\section{Le label : Gage de confiance de la marque}

Selon Chameroy et Chandon (2010), " l'étiquette est un signe distinctif de la marque, symbolisant l'une des dimensions intrinsèques du produit, émanant d'un tiers ou perçu comme tel ».

Sur le même sujet, Sauvee et Valceschini (2003) attestent que les critères de marque de type « label » prennent généralement en compte les améliorations scientifiques et techniques et 
l'amélioration du niveau des produits actuels afin de maintenir un écart avec eux ». Contribuer à améliorer la perception de la marque et principalement dans le cadre de produits coopératifs élaborés à partir de produits locaux.

La valeur économique du signal vient de sa pertinence et de sa crédibilité Sauvee et Valceschini (2003). En effet, les certifications et labels contribuent à augmenter la crédibilité des produits et donc la confiance. Cependant, la crédibilité du signal peut être représentée comme une arme à double tranchant dans la mesure où une mauvaise perception impactera sa réputation Valceschini et Mazé (2000).

Dans le contexte particulier de la coopérative, la notion de labels a été mise en avant dans la politique de produits et de communication de la coopérative. Une étude exploratoire a été réalisée par Ghilane et Aomari (2018), pour explorer la stratégie marketing et branding de la coopérative marocaine qui internationalise sa marque. Les résultats ont montré que la qualité, les labels et les certifications étaient les variables les plus utilisées dans l'internationalisation de la marque.

Depuis l'entrée en vigueur de la loi 25-06 relative aux Signes Distinctifs d'Origine et de Qualité (SDOQ), plusieurs produits ont été étiquetés selon l'appellation d'indication géographique (IG), l'appellation d'origine protégée. (AOP), et le label agricole (LA). L'Organisation des Nations Unies pour l'alimentation et l'agriculture (FAO) présente l'indication géographique comme une attribution du critère de qualité ou de réputation à une origine géographique. Pour l'appellation d'origine protégée, il s'agit d'une forme plus développée que l'indication géographique qui prend en compte les facteurs naturels, les caractéristiques du produit et le facteur humain. Pour l'étiquetage agricole, il s'agit de la reconnaissance qu'un produit a un certain niveau de qualité supérieur à celui existant.

Atkinson et Rosenthal (2014) ont mené une étude sur la confiance et l'attitude des consommateurs envers la certification en tenant compte du niveau d'implication des consommateurs et du niveau de détail des informations.

Les résultats de l'étude montrent que les labels les plus détaillés et les plus significatifs impactent positivement la confiance des consommateurs, au détriment du niveau d'implication.

Dans les relations consommateur - marque, la confiance et surtout la confiance initiale représente la première étape du processus relationnel. Développer une relation de confiance entre le consommateur et la marque conduira à des relations plus profondes telles que la satisfaction, l'attachement, l'engagement pour conduire à la fidélité.

\section{Méthodologie de recherche}

Sur la base des conclusions tirées de la littérature, les labels participent au renforcement de la confiance en la marque et donc au développement des relations marque - consommateur. Dans cet article, nous étudierons l'impact du label dans l'internationalisation de la marque coopérative marocaine.

Selon Gurviez et Ebende Kouedi (2013), la variable labels est représentée dans le contexte des facteurs liés aux « tiers » avec la «bouche à oreille » et le témoignage en ligne. Les facteurs « tiers » participent au développement de la confiance initiale dans une nouvelle marque 
implantée et se traduisent en intention comportementale représentée par l'intention d'achat et l'intention de recommander.

Figure 1 : Modèle conceptuel de l'impact des labels sur la marque

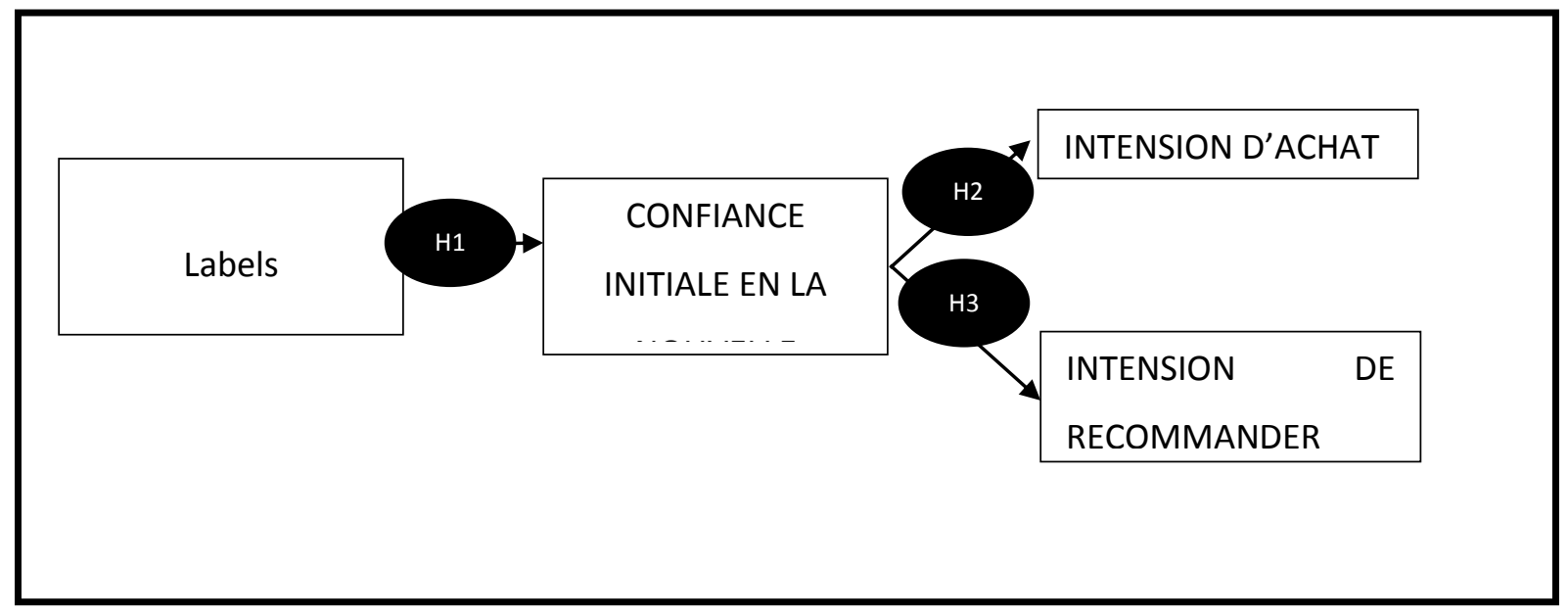

Source : Réalisé par l'auteur

Selon Sauve et Valceschini (2003) la valeur économique du signal dépend de la pertinence et de la crédibilité de celui-ci. Les certifications et les labels contribuent à soutenir l'image perçue de la qualité en créant plus de crédibilité et de confiance. De là, nous soulignons l'hypothèse (H1) qui énonce que la présence de certification/labels augmente la confiance initiale dans la marque.

La littérature atteste que la confiance initiale impacte l'intention d'achat et de recommandation. Selon Kennedy et al. (2001) c'est dans la phase d'établissement que le consommateur recherche des informations, effectue l'achat initial et entame le processus de construction de la confiance, (H2), l'intention d'achat de la nouvelle marque est impactée positivement par la confiance initiale. Selon McKnight et al. (2002), Wakefield et al. (2004) et Chouk (2005), une influence positive de la confiance initiale dans un vendeur en ligne sur l'intention d'acheter sur le site de ce vendeur et l'intention de recommander le site de ce vendeur à l'entourage. (H3) L'intention de recommander est influencée positivement par la confiance initiale dans la nouvelle marque.

Pour tester le modèle, nous avons opté pour la méthode de processus d'opérationnalisation.

Concernant l'évaluation de la confiance que les consommateurs accordent aux labels, nous avons tenu à utiliser l'échelle proposée et validée par Gurviez et Korchia (2002), qui visait à tester la confiance des consommateurs dans les marques. Cette échelle a été adaptée par Bachelor et Gobet (2014) dans leur étude des connaissances et de la confiance dans les étiquettes.

Nous nous sommes donc basés sur les éléments évaluant la confiance dans le label tout en nous adaptant au contexte de la coopérative marocaine travaillant dans les produits locaux. L'évaluation est faite sur la base de l'échelle Likert à 5 niveaux.

La mesure de l'intention d'achat selon Kumar et al. (2009) englobe 2 items : « J'ai l'intention d'acheter fréquemment des marques de produits locaux des coopératives marocaines. » Et « J'envisage d'acheter plus souvent des marques de produits locaux des coopératives marocaines. Aussi, Kim et al (2012) proposent 3 autres items pour évaluer l'intention d'achat. 
A titre d'intention de recommander, nous retenons 4 items utilisés dans les travaux de Moon et Kim (2001); Hsu et Lin (2008); Ahn et al. (2007) que nous avons adaptés à notre contexte.

L'étude a porté sur un échantillon de 200 personnes choisies par la méthode de convenance. L'échantillon est composé de citoyens français. Le choix de la population se justifie par le fait que la majorité des coopératives citent la France comme pays de destination de leurs produits, et d'autre part, la facilité d'accès à la cible en plus des considérations de proximité culturelle.

Pour le traitement des données, nous avons d'abord établi l'analyse factorielle exploratoire qui a validé le pré-test, puis nous avons procédé dans un second temps à l'élaboration de l'analyse confirmatoire. La validation du modèle est faite par la méthode LISREL.

\section{Résultats et discussion :}

La validation des items combine deux techniques : le recueil des retours sur le questionnaire et une analyse par la composante principale où nous avons mobilisé des indicateurs comme la rotation varimax, l'indice KMO et le test de Bartlett.

Pour l'axe relatif à la confiance dans l'étiquette, l'analyse rotationnelle indique que tous les items doivent être pris en considération. Cependant, puisque notre sujet porte sur la confiance initiale, nous supprimerons les items relatifs à la bienveillance, étant donné que la littérature indique que la confiance initiale est principalement mesurée par des items relatifs à l'intégrité et à la crédibilité.

L'objectif de l'analyse de l'indice KMO et du test de sphéricité de Bartlett est de mesurer l'adéquation de l'échantillonnage et si la matrice est une matrice d'identité.

L'indice KMO des variables : Confiance dans les étiquettes, Intention de recommander, Intention d'achat, est supérieur à 0,6 . Cela nous indique que les corrélations entre les items sont de bonne qualité pour tous ces facteurs.

Le résultat du test de sphéricité de Bartlett est significatif $(\mathrm{p}<0,05)$ pour toutes les variables. On peut donc rejeter l'hypothèse nulle que nos données proviennent d'une population dont la matrice est une matrice identité. Les corrélations ne sont donc pas toutes égales à zéro. On peut donc continuer l'analyse.

L'analyse de la fiabilité des échelles de mesure a été réalisée à l'aide de l'indicateur Alpha de Cronbach. Selon Nunally (1978), une valeur supérieure ou égale à 0,70 est généralement considérée comme un critère pour démontrer la cohérence interne d'une échelle de mesure. Les résultats montrent que tous les facteurs ont une valeur supérieure à 0,7 ce qui implique une cohérence interne dans les échelles de mesure.

La modélisation par équations structurelles représente « une approche statistique globale permettant de tester des hypothèses portant sur les relations entre variables observées et variables latentes » Hoyle (1995). Les résultats du test de lien de causalité et la validation des hypothèses ont permis de constater que la variable « Label » a un impact positif et significatif sur la variable « Confiance Initiale » $(\mathrm{Reg}=$ $0,663 ; \mathrm{T}$ de Student $=9,045 ; \mathrm{p}$-value $=0,000)$. De plus, la variable «Confiance initiale $»$ a un impact positif et significatif sur la variable «Intention d'achat $(\operatorname{Reg}=0,651 ; \mathrm{T}$ de l'étudiant $=6,717$; valeur $\mathrm{p}=0,000$ et sur la variable «Intention de recommander $\gg(\operatorname{Reg}=0,584 ; \mathrm{T}$ de Student $=6,389$; valeur $\mathrm{p}=0,000)$.

L'hypothèse que nous avons développée dans le cadre des facteurs « tiers » du modèle présenté par Gurviez et Ebende Kouedi (2013) porte sur les labels et leur impact sur la confiance initiale. Nous vérifions cette hypothèse dans la présente étude. Si la notoriété n'influence pas la confiance initiale dans la marque de la coopérative marocaine œuvrant dans les produits locaux, le label joue un rôle plus 
important. Certaines personnes interrogées ont exprimé : « Je suis particulièrement attachée à l'équité des produits que j'achète »; " Pour moi, le plus important est le label (encore plus que bio) lorsque j'achète des produits du Maroc. », Et un autre répondant a suggéré qu'un « Label Reconnu, améliorer la transparence de leur marque (process de fabrication, ingrédients, étiquette) ».

Ces réponses ouvertes permettent de démontrer d'une part l'importance accordée aux labels, et d'autre part d'expliquer en partie le rejet de l'impact de la réputation sur la confiance initiale, dans la mesure où les consommateurs ont besoin de preuves, puis de labels.

Nous avons évoqué, dans notre partie théorique, les travaux d'Atkinson et Rosenthal (2014) qui ont mené une étude sur la confiance et l'attitude des consommateurs vis-à-vis de la certification en tenant compte du niveau d'implication des consommateurs.

Les résultats de l'étude montrent que les étiquettes les plus détaillées et les plus significatives ont un impact positif sur la confiance des consommateurs, ce qui correspond parfaitement aux résultats que nous avons trouvés.

\section{Conclusion :}

Le processus d'internationalisation de la marque est continu et étroitement lié à une stratégie de mixmarketing Dinnie (2011), axée sur la création d'associations fortes, solidaires et uniques dans l'esprit du consommateur Keller (2008). Le marketing mix est un facteur essentiel dans la prise de décision sur les marchés et combine des éléments qui sont liés et qui peuvent être influencés.

Les décisions prises dans chaque élément marketing influencent les dimensions de la valeur de la marque Yoo, et al. (2000). En fait, le marketing mix illustre la direction de la marque Aghaei et al. (2014).

Ainsi, on note le lien fort entre la décision d'internationalisation, le mix marketing, et la marque pour développer une stratégie de marque internationale cohérente.

Dans le contexte de la coopérative et principalement de la coopérative marocaine, des études ont souligné que les variables les plus utilisées et exigées du marché pour développer une stratégie de marque forte sont la qualité et les labels. Fondamentalement, la littérature a identifié les deux comme des antécédents qui ont un impact sur la confiance initiale. Cependant, dans notre étude liée au cas des marques coopératives marocaines, le consommateur français potentiel ne tient pas compte de la réputation, mais prend en considération des preuves réelles telles que l'étiquetage.

Dans ce cas, la coopérative orientera sa stratégie de marque en l'accompagnant de certifications et de labels plutôt que de se baser sur une notoriété non prise en compte par le marché.

Nous soulignons parmi les limites de la recherche l'élargissement de l'échantillon à la fois qualitativement et quantitativement. Aussi, la recherche porte sur l'acceptation de la marque à travers la variable de « confiance ». Il sera intéressant d'étudier d'autres niveaux de relations comme l'engagement et la fidélité.

\section{BIBLIOGRAPHIE}

[1] Atkinson, L., \& Rosenthal, S. (2014). Signaling the Green Sell: The Influence of Eco-Label Source, Argument Specificity, and Product Involvement on Consumer Trust. Journal of Advertising, 43 (1), 33-45.

[2] Attouch, H. (2014). Pratiques du marketing coopératif : cas de la COPAG. REMACOOP, 4, 6- 13. 
[3] Beverland,M.(2007).Can cooperatives brand? Exploring the interplay between cooperative structure and sustained brand marketing success. Food Policy,32(4),480-495.https://doi.org/10.1016/j.foodpol.2006.10.004

[4] Boisvert, J. (1981). Le marketing dans la perspective coopérative. La gestion moderne des coopératives, 117-151.

[5] Chameroy, F. (2013). Les effets du label sur la qualité perçue, les relations à la marque et le consentement à payer, Thèse de Doctorat en Sciences de Gestion, IAE d'Aix-en-provence, Université d'Aix-Marseille.

[6] Cook, M. L., \& Iliopoulos, C. (1999). Beginning to Inform the Theory of the Cooperative Firm: Emergence of the New Generation Cooperative. The Finnish Journal of Business Economics (4), 525-535.

[7] Ebendé Kouedi, S., \& Gurviez, P. (2013). Le rôle de la confiance initiale dans la phase d'etablissement de la relation consommateur-marque : proposition d'un cadre conceptuel preliminaire. Association Française de Marketing, 1-22.

[8] Errasti, A., Heras, I., Bakaikoa, B., \& Elgoibar, P. (2003). The internationalisation of cooperatives: the case of the mondragon cooperative corporation. Annals of public and cooperative economics, 74 (4), 553-584.

[9] Fort,F.,\&Fort,F.(2006).Alternatives marketing pour les produits de terroir. Revue Française de Gestion, 32(162), 145160. https://doi.org/10.3166/rfg.162.145-160

[10] Michel, G. (2009). Au coeur de la marque Les clés du management des marques . 2e édition, Etude (broché).

[11] Prévost, P. (2001). « La formulation de stratégies coopératives et le développement du milieu» Sherbrooke, IRECUS, Cahiers de recherche IREC 01-08.

[12] Rastoin, J. (2004). Quelle stratégie pour les produits de Terroir dans un contexte de globalisation des marchés? Séance Du 06 Mai 2004, Cycle Comprendre Les Agricultures Du monde d'après les cafés-débats à Marciac

[13] Sauvée,L., \& Valceschini,E. (2003). Agro-alimentaire : la qualité au cœur des relations entre agriculteurs, industriels et distributeurs. Déméter, (May2014), 181-226.

[14] Ghilane Hind and Aomari Amina (2018), «Initial Confidence: Determining Factors for the Internationalization of the Moroccan Cooperative Brand» Journal of Marketing Research and Case Studies, Vol. 2018 (2018), Article ID 244080, DOI: $10.5171 / 2018.244080$

[15] Gouin, S. \& Perraud, C. (2008). Coopératives de sel littoral atlantique quelles réflexions stratégiques produit Colloque SFER les entreprises coopératives agricoles, mutation et perspectives. Paris.

[16] Grandval, S. \& Soparnot R. (2005). Le développement durable comme positionnement stratégique. in Wolf D, Mauléon F, Le management durable l'essentiel du développement durable appliqué aux entreprises, Hermes Science Publications, 252.

[17] Gurviez, \& Korchia. (2002). Proposition d'une échelle de mesure multidimensionnelle de la confiance dans la marque. Recherches et Application en Marketing , 17 (3), 41-61.

[18] Wakefield, R., Stocks, M., \& Wilder, W. (2004). The role of web site characteristics in initial trust formation. Journal of Computer Information Systems , 45, 94-103. 